\title{
Chronic Endometritis: Prevalence-using Hysteroscopy and Cd138 Immunohistochemistry- and Impact on Reproductive Outcome in Patients with Previous IVF/ICSI Failure
}

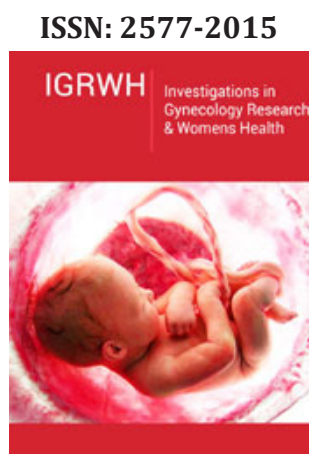

*Corresponding author: Mahmoud HM, Department of Obstetrics/Gynecology, Faculty of Medicine, University of Alexandria, Egypt

Submission: 策June 15, 2019

Published: 海June 26, 2019

Volume 3 - Issue 1

How to cite this article: Mahmoud H, Hebisha S. Chronic Endometritis: Prevalence-using Hysteroscopy and Cd138 Immunohistochemistry-and Impact on Reproductive Outcome in Patients with Previous IVF/ICSI Failure. Invest Gynecol Res Women's Health.3(1) IGRWH.000552.2019.

DOI: 10.31031/IGRWH.2019.03.000552

Copyright@ Mahmoud HM, This article is distributed under the terms of the Creative Commons Attribution 4.0 International License, which permits unrestricted use and redistribution provided that the original author and source are credited.

\section{Mahmoud HM* and Hebisha SA}

Department of Obstetrics/Gynecology, Faculty of Medicine, University of Alexandria, Egypt

\begin{abstract}
Background: A healthy receptive endometrium is essential for embryo implantation. Recently, the relationship between chronic endometritis (CE) and infertility-related conditions such as previous implantation failure has emerged as an area of inquiry.
\end{abstract}

Objective: To evaluate the prevalence of CE in women with previous IVF/ICSI failure using hysteroscopy and CD138 immunohistochemistry and to determine whether subsequent antibiotic treatment positively impacts outcome in these cases.

Design: prospective cohort study.

Setting: El-Shat by Maternity University Hospital.

Materials and methods: 145 women with history of previous IVF/ICSI failure underwent hysteroscopy and endometrial sampling for CD138 immunohistochemistry detection. Patients were placed in two groups based on presence or absence of CE (group 1 and 2 respectively). Patients in group 1 underwent treatment with oral antibiotics for at least 3 weeks until repeat follicular phase biopsy showed no CE. Primary outcome was sensitivity, specificity and accuracy of hysteroscopy in relation to immunohistochemistry, (as a gold standard) in diagnosis of CE. Secondary outcomes were implantation and pregnancy rates in the post-treatment ICSI attempt.

Result: Prevalence of CE was 23.4\% (34/145) using hysteroscopy and 19.3\% (28/145) using immunohistochemistry (golden standard) showing $78.6 \%, 89.7 \%$, and $87.5 \%$ sensitivity, specificity and accuracy respectively. Implantation rate in subsequent cycles was significantly higher in group A vs. B ( $35.16 \%$ vs $16.43 \%, \mathrm{p}=0.001 *$ ). Group A also showed higher pregnancy rate-yet not reaching statistical significance-compared to group B $(57.14 \%$ vs $34.18 \%$, $\mathrm{p}=0.076)$.

Conclusion: In women with previous IVF/ICSI failure, hysteroscopy is a reliable method for diagnosing CE. The successful treatment of CE significantly improves implantation and pregnancy rates in subsequent attempts. Confirmation of cure is essential before new trials.

Keywords: Chronic endometritis; Immunohistochemistry; Hysteroscopy; IVF/ICSI outcome; Antibiotic treatment; Implantation failure

\section{Introduction}

To date, millions of couples have overcome fertility problems using IVF cycles. Although it continues to provide hope for lots of infertile couples, yet failure-and more essentially repeated failure-of this expensive treatment modality is very frustrating for both the infertile couple and the treating doctor. Failure of implantation can be caused by factors related to the embryo itself or to the endometrial receptivity. The latter can be altered by multiple lesions such as adhesions, thin endometrium, submucous myomas, septa, and polypi [1,2]. There is no consensus on the repeated implantation failure (RIF) definition, however, failure to achieve pregnancy after two or more embryo transfers or after transfer of 10 or more embryos with good quality is- for the lack of a more accurate one-accepted as a definition [3]. Contrary to those causes that can easily be diagnosed by ultrasound examination, chronic endometritis (CE) is a special cause that can lead to repeated implantation failure through decreasing the endometrial receptivity and is trickier to diagnose [4]. Chronic endometritis 
is found in $0.2-46 \%$ of infertile women $[4,5]$. Studies showed that its prevalence is between $14-60 \%$ in cases with RIF [6-9]. And while [7] demonstrated that the implantation rate is lower in the CE patients in the IVF cycle following treatment compared to IVF cycles in women who are CE negative [7,8]. Cicinelli et al. [9] showed that adequate antibiotic treatment in RIF patients with CE significantly improved the pregnancy rate in comparison to those patients with persistent CE [9]. These high numbers and reports cannot be ignored when dealing with ART patients in general and more so when dealing with problems that still have no definite protocols for management as RIF patients.

The problem with CE is that it is mostly an asymptomatic condition and when it is symptomatic, the symptoms are very nonspecific like pelvic pain, abnormal bleeding, or excessive discharge, thus it can neither be diagnosed clinically nor with ultrasound examination, hence it can easily be missed [10]. During hysteroscopy, finding of stromal edema, micro-polypi, and hyperemia whether diffuse or focal raise suspicion of CE [11,12]. Endometrial biopsy remains the definitive diagnostic tool for $\mathrm{CE}$. Among the different leukocytes that infiltrate the endometrium in cases of chronic endometritis, the diagnosis depends on finding plasma cells in the endometrial stoma [4]. Unfortunately, the histopathological diagnosis of CE is tedious and with low accuracy. The results can be affected by the phase of the menstrual cycle, infiltration with other inflammatory cells that are difficult to distinguish from plasma cells, or by proliferation of the stroma $[13,14]$.

Transmembrane heparin sulfate proteoglycan syndecan-1 (CD138) is a plasma cell-specific marker that can be detected by immunohistochemistry and used to improve the detection rate of CE $[15,16]$. The objective of the present study was to evaluate the prevalence of chronic endometritis in women with previous IVF/ ICSI failure using different diagnostic modalities and to determine the effect of treatment on the success rates of the subsequent trials.

\section{Material and Methods}

145 women with previous IVF/ICSI failure who were planning for another trial were recruited in this study. The study was approved by the ethics committee of the Faculty of Medicine, university of Alexandria. All couples included in the study signed an informed consent before inclusion in the study.

\section{Inclusion criteria}

i. Age below 40 years

ii. Absence of uterine cavity abnormality by previous HSG or ultrasonography.

\section{Hysteroscopy and biopsy}

All patients underwent hysteroscopy for detection of any signs of chronic endometritis; initial evaluation of the cavity was performed using panoramic view followed by meticulous search for signs such as micro-polypi, edema or hyperemia, and accordingly patients were classified to patients with and without signs of CE on hysteroscopy. Afterwards, an endometrial sample was obtained for CD138 immunohistochemistry.

\section{Immunohistochemistry (IHC)}

Immediately after collection, the endometrial sample was fixed in $10 \%$ neutral formaldehyde then sent to the pathology lab, there, biopsies were immersed in paraffin then serial micro-sections were cut. Slides were dewaxed in preparation for immunohistochemistry staining with plasma cell specific CD138 monoclonal antibodies. Cases were considered CE positive if five or more plasma cells were observed per high power field (x400 magnification). According to the CD138 immunohistochemistry results, patients were divided into 2 groups: Group 1 patients with CE diagnosed by positive CD138 testing, and group 2 where CD138 was negative and thus were diagnosed as negative for chronic endometritis.

Patients in group 1 (CE positive) received oral treatment in the form of doxycycline $100 \mathrm{mg}$ twice daily for 21 days. Afterwards a repeat endometrial sample was obtained using a pipelle (Rocket Endometrial Sampling Syringe, Rocket Medical) for testing and cases still positive for CD138 received a 14-day oral course of ciprofloxacin $500 \mathrm{mg}$ twice per day in combination with metronidazole $500 \mathrm{mg}$ twice per day $[17,18]$.

\section{ICSI cycles}

All cases underwent ICSI cycles using either the long agonist protocol or the antagonist protocol according to the decision made by their treating doctor based on their age, ovarian reserve tests and previous oocyte yield.

All cases underwent standard ICSI procedure and embryo transfer was performed either on day 3 or day 5 according to the number and quality of the embryos.

Both groups were compared as regards the accuracy of the hysteroscopic findings of chronic endometritis in comparison to the IHC finding of CD138 (as a gold standard for diagnosis of CE). Both groups were also compared as regards the implantation rate and the clinical pregnancy rate as secondary outcomes where the clinical pregnancy was defined as demonstration of an intrauterine gestational sac with a positive heartbeat 4 weeks after embryo transfer.

\section{Results}

As shown in Table 1, the prevalence of chronic endometritis was $23.4 \%$ by hysteroscopy while it was only $19.31 \%$ using CD138 immunohistochemistry staining. Of the 28 CD138 positive cases, only 22 showed positive hysteroscopic signs. On the other hand, 12 cases that showed positive hysteroscopic signs of $\mathrm{CE}$ were CD138 negative. Sensitivity of hysteroscopy in diagnosing CE was $78.6 \%$, specificity was $89.7 \%$, accuracy was $87.5 \%$, and negative predictive value was $94.6 \%$. As shown in Figure 1, out of the 145 women included in this study 5 did not undergo embryo transfer; 2 due to fear of ovarian hyperstimulation syndrome (OHSS), 2 due to poor endometrium (all 4 cases had all their embryos vitrified) and one case had cancelled embryo transfer due to very poor embryo quality. The implantation rate was significantly higher in group 1 cases (treated CD138 positive cases) than in group 2 cases (CD138 negative cases) (35.16\% vs $\left.16.43 \%, \mathrm{p}=0.001^{*}\right)$. As 
regards the pregnancy rate, there was a marked trend towards a higher pregnancy rate in group 1 than in group 2 \{57.14\%(16/28) vs $34.18 \%(40 / 117)\}$. This trend, however, did not reach statistical significance $(\mathrm{p}=0.076)$ (Table 2).

Table 1: Sensitivity, specificity and accuracy of hysteroscopy in relation to CD138 (as a gold standard) in diagnosis of CE.

\begin{tabular}{|c|c|c|c|c|}
\hline & & \multicolumn{2}{|c|}{ CD138 } & \multirow{3}{*}{ Total } \\
\hline & & -ve & $+v e$ & \\
\hline & & $117(80.6 \%)$ & $28(19.31 \%)$ & \\
\hline \multirow{4}{*}{ signs of CE at hysteroscopy } & \multirow{2}{*}{-ve } & 105 & 6 & \multirow{2}{*}{$111(76.6 \%)$} \\
\hline & & (True negative) & (False negative) & \\
\hline & \multirow{2}{*}{$+\mathrm{ve}$} & 12 & 22 & \multirow{2}{*}{$34(23.4 \%)$} \\
\hline & & (False positive) & (True positive) & \\
\hline \multirow{2}{*}{\multicolumn{2}{|c|}{ Total }} & 117 & 28 & \multirow{2}{*}{145} \\
\hline & & Gold standard & Gold standard & \\
\hline \multicolumn{2}{|c|}{ Sensitivity of hysteroscopy } & $78.60 \%$ & & \\
\hline \multicolumn{2}{|c|}{ Specificity of hysteroscopy } & $89.70 \%$ & & \\
\hline \multicolumn{2}{|c|}{ Accuracy of hysteroscopy } & $87.50 \%$ & & \\
\hline \multicolumn{2}{|c|}{ Negative predictive value } & $94.60 \%$ & & \\
\hline
\end{tabular}

Table 2: Distribution of the studied cases according to immunohistochemistry and correlation with implantation rate and pregnancy outcome.

\begin{tabular}{|c|c|c|c|c|}
\hline & Group 1 & Group 2 & \multirow{2}{*}{ Total } & \\
\hline & CD 138 Positive & CD 138 Negative & & \\
\hline Implantation rate & $35.16 \%$ & $16.43 \%$ & & $0.001^{*}$ \\
\hline \multirow{3}{*}{ Pregnant } & 16 & 40 & 56 & $X^{2}=5.17$ \\
\hline & $57.14 \%$ & $34.18 \%$ & $38.62 \%$ & $\mathrm{Df}=2$ \\
\hline & & & & $\mathrm{P}=0.076$ \\
\hline \multirow{2}{*}{ Not } & 11 & 73 & 84 & \\
\hline & $39.28 \%$ & $62.39 \%$ & $57.93 \%$ & \\
\hline \multirow[t]{2}{*}{ Cancelled } & 1 & 4 & \multirow{2}{*}{5} & \\
\hline & $3.57 \%$ & $3.41 \%$ & & \\
\hline Total & 28 & 117 & 145 & \\
\hline
\end{tabular}
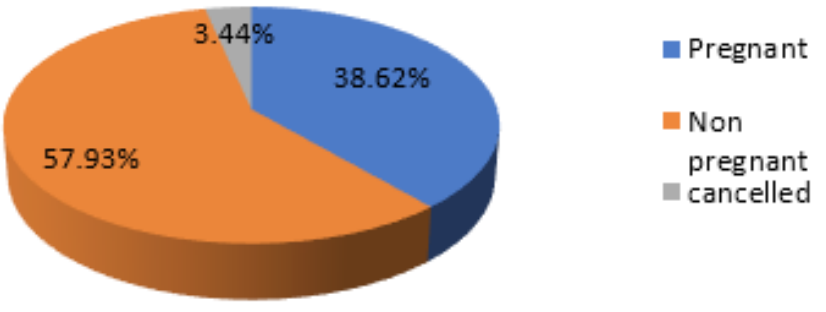

Figure 1: Distribution of the studied group according to pregnancy rate.

\section{Discussion}

Successful implantation in IVF requires the co-existence of multiple factors namely a normal uterine cavity, a proper immune system, a receptive endometrium, and a reasonably good quality embryo $[19,20]$. One of the causes that might affect the endometrial receptivity and hence lead to implantation failure is chronic endometritis which is defined as "chronic inflammation of the endometrium [21]. The main problem with CE is the difficulty of its diagnosis [4]. In addition to the lack of specific symptoms, the hysteroscopic diagnosis depends on the clinician's experience and the gold standard for diagnosis rests on the identification of plasma cells in the endometrial stroma [11,12]. Unfortunately, even histologic identification of plasma cells with HE staining is not without limitations: besides the technical problems of inadequate staining and bad endometrial sample preservation, proliferation of the stromal cells as well as excessive mitosis may mask the plasma cells and lead to false negative diagnosis, on the other hand, false positive diagnosis is reported since infiltrates with mononuclear cells or plasmacytoid stromal cells may mimic plasma cells [15,22]. Syndecan-1 (CD138) is a plasma cell specific marker that can be 
detected using immunohistochemistry staining with monoclonal antibodies. The use of CD138 IHC staining improved the sensitivity and specificity of plasma cell detection in comparison to HE and HPS staining [23,24]. Furthermore, it minimized the variability between observers and shortened the lab time for reaching a decision $[15,25,26]$. The present study found nearly similar prevalence of CE in women with previous IVF/ICSI failure using hysteroscopy and IHC staining (23.4\% and $21.4 \%$ ). 12 of the cases that showed signs of CE on hysteroscopy were CD138 negative while only 6 of the CD138 positive cases showed no signs of CE in hysteroscopy. A possible explanation for the lower prevalence of CE on IHC staining was provided by Yang et al stating that sampling with a pipelle does not adequately represent the whole endometrium [27]. A hysteroscopic guided biopsy using grasping forceps from focal areas of suspicion might improve the diagnostic accuracy of IHC staining. The present study found the sensitivity of hysteroscopy in diagnosis of CE to be $78.6 \%$, its specificity $89.7 \%$ the diagnostic accuracy was $87.5 \%$, and the negative predictive value was $94.6 \%$.

As mentioned before, multiple authors have found the prevalence of CE in RIF patients to range from 14-60\% [6-9]. This can be explained by the fact that there is no universal consensus on the criteria for diagnosing $\mathrm{CE}$ and different authors used different cut off numbers of plasma cells to confirm the diagnosis. Furthermore, Adegboyega et al. [14] suggested that any variations in the rate of diagnosis of CE between histology and hysteroscopy might be due to the limitations of the former method. Bouet et al. [6] reported nearly identical prevalence in women with poor reproductive outcome (RIF and repeated pregnancy loss RPL), they reported positive CE signs in hysteroscopy in $24 \%$ of cases, while histologically confirmed CE was found in $21.3 \%$ of cases (14\% in RIF and 27\% in RPL). Similarly, in the study by Zargar et al. [28]. The prevalence of CE in RIF patients was $23.4 \%$ by IHC and $21.3 \%$ by hysteroscopy [28]. Bouet et al. [6] found the sensitivity of hysteroscopy to be only $40 \%$ while the specificity was $80 \%$.

Similar to our results in multiple studies $[9,11,12]$ reported a diagnostic accuracy of $93.45 \%$, [28] demonstrated sensitivity, specificity, and negative predictive value of $86.2 \%, 87.3 \%$ and $94.8 \%$ respectively, and Polisseni et al. [29] showed $93.2 \%$ specificity with a high negative predictive value. In Contrast, Yang et al. [27] found lower sensitivity and specificity of hysteroscopy (35.25 and 67.5\%) respectively [27] and Song et al. [30] reported that the diagnostic accuracy of hysteroscopy was only $67 \%$ and they concluded that hysteroscopy should not replace histologic examination [30]. In this study, the implantation rate and the clinical pregnancy rates were higher in women with treated CE than in women with negative CE $(35.16 \%$ vs $16.43 \%$ and $57.14 \%$ vs $34.18 \%$ respectively). This denotes that CE has a negative impact on the success of IVF/ICSI and its treatment dramatically improves the outcome, while cases negative for CE had probably other undiagnosed causes for their previous failure, hence the outcomes of the following trials were not markedly affected. Likewise, Kitaya et al. [17] demonstrated a live birth rate of $32.8 \%$ in the first cycle in cured RIF/CE cases which was significantly higher than the $22.1 \%$ reached in the RIF/ CE negative cases [31].
Multiple other clinical studies showed that treatment of CE could produce dramatic improvements in future outcomes [9,18,27]. In their systematic review and meta-analysis, [32] demonstrated that the implantation rate (IR), the clinical pregnancy rate (CPR), the ongoing pregnancy rate (OPR) and the live birth rate (LBR) did not show any significant difference between women with CE receiving antibiotics (without Histologic confirmation of cure) from untreated controls. All the outcomes were higher in women with confirmed cure [32]. Similarly, in the review by Kimura et al. [33] the IR, CPR and OPR significantly improved after cure of CE [33]. Furthermore, [9] in a retrospective study on women with RPL- found a higher LBR in women with normalized hysteroscopic findings after treatment than in women with persistent signs regardless of the findings of microbial culture [34]. In contrast to these findings, Kasius et al. [4] found no significant difference in the CPR and LBR between CE positive and CE negative patients [4]. Johnston et al. [7] observed a low implantation rate in successfully treated CE positive cases. They suggested that the initial inflammatory process might have altered the internal uterine environment resulting in a persistently low endometrial receptivity [7].

\section{Conclusion}

Chronic endometritis is an important cause for implantation failure. Hysteroscopy is a reliable method for diagnosis of chronic endometritis with an especially high specificity, diagnostic accuracy, and negative predictive value. Treatment of CE using oral antibiotics significantly improves the outcome of the following ICSI cycles. In case of persistent CE after initial treatment course, second line antibiotics should be administered, and confirmation of cure is essential before embarking on subsequent ICSI cycles.

\section{References}

1. Taylor E, Gomel V (2008) The uterus and fertility. Fertil Steril 89(1): 1-16.

2. Bosteels J, Weyers S, Puttemans P, Panayotidis C, Herendael BV, et al. (2010) The effectiveness of hysteroscopy in improving pregnancy rates in subfertile women without other gynaecological symptoms: a systematic review. Hum Reprod Update 16(1): 1-11.

3. Eltoukhy T, Taranissi M (2006) Towards better quality research in recurrent implantation failure: standardizing its definition is the first step. Reprod Biomed Online 12(3): 383-385.

4. Kasius C, Fatemi H, Bourgain C, Sie GD, Eijkemans R, et al. (2011) The impact of chronic endometritis on reproductive outcome. Fertil Steril 96(6): 1451-1456.

5. Carvalho FM, Aguiar FN, Tomioka R, de Oliveira RM, Frantz N, et al. (2013) Functional endometrial polyps in infertile asymptomatic patients: a possible evolution of vascular changes secondary to endometritis. Eur J Obstet Gynecol Reprod Biol 170(1): 152-156.

6. Bouet PE, Hachem HE, Monceau E, Gariepy G, Kadoch IJ, et al. (2016) Chronic endometritis in women with recurrent pregnancy loss and recurrent implantation failure: prevalence and role of office hysteroscopy and immunohistochemistry in diagnosis. Fertil Steril 105(1): 106-110.

7. Ananny EBJ, Hartnett J, Engmann LL, Nulsen JC, Sanders MM, et al. (2010) Chronic endometritis is a frequent finding in women with recurrent implantation failure after in vitro fertilization. Fertil Steril 93(2): 437-441.

8. Conway DA, Ketefian A, Shamonki M (2010) Chronic endometritis: A common finding in good prognosis patients with failed implantation following in vitro fertilization. Fertil Steril 94(4): S175. 
9. Cicinelli E, Matteo M, Tinelli R, Lepera A, Alfonso R, et al. (2015) Prevalence of chronic endometritis in repeated unexplained implantation failure and the IVF success rate after antibiotic therapy. Hum Reprod 30(2): 323-330.

10. Romero R, Espinoza J, Mazor M (2004) Can endometrial infection/ inflammation explain implantation failure, spontaneous abortion, and preterm birth after in vitro fertilization? Fertil Steril 82(4): 799-804.

11. Cicinelli E, Resta L, Nicoletti R, Zappimbulso V, Tartagni M, et al. (2005) Endometrial micropolyps at fluid hysteroscopy suggest the existence of chronic endometritis. Hum Reprod 20(5): 1386-1389.

12. Cicinelli E, Ziegler DD, Nicoletti R, Colafiglio G, Saliani N, et al. (2008) Chronic endometritis: correlation among hysteroscopic, histologic, and bacteriologic findings in a prospective trial with 2190 consecutive office hysteroscopies. Fertil Steril 89(3): 677-684.

13. Kasius JC, Broekmans FJ, Sie DM, Bourgain C, Eijkemans MJ, et al. (2012) The reliability of the histological diagnosis of endometritis in asymptomatic IVF cases: a multicenter observer study. Hum Reprod 27(1): 153-158.

14. Adegboyega PA, Pei Y, Mclarty J (2010) Relationship between eosinophils and chronic endometritis. Hum Pathol 41(1): 33-37.

15. Garner IBB, Nickell JA, Korourian S (2004) Routine syndecan-1 immunohistochemistry aids in the diagnosis of chronic endometritis. Arch Pathol Lab Med 128(9): 1000-1003.

16. Chen YQ, Fang RL, Luo YN, Luo CQ (2016) Analysis of the diagnostic value of CD138 for chronic endometritis, the risk factors for the pathogenesis of chronic endometritis and the effect of chronic endometritis on pregnancy: a cohort study. BMC Womens Health 16(1): 60.

17. Kitaya K, Tada Y, Taguchi S, Funabiki M, Hayashi T, et al. (2012) Local mononuclear cell infiltrates in infertile patients with endometrial macropolyps versus micropolyps. Hum Reprod 27(12): 3474-3480.

18. Queen DB, Bernardi LA, Stephenson MD (2014) Chronic endometritis in women with recurrent early pregnancy loss and/or fetal demise. Fertil Steril 101(4): 1026-1030.

19. Margalioth EJ, Chetrit AB, Gal M, Geva TE (2006) Investigation and treatment of repeated implantation failure following IVF-ET. Hum Reprod 12: 3036-3043.

20. Simon A, Laufer N (2012) Repeated implantation failure: clinical approach. Fertil Steril 97(5): 1039-1043.

21. Neelam P, Vitthala S (2001) Are the causes of recurrent implantation failure a myth evidence-based reality? Fertil Steril 96(3): S279.

22. Garner IBB, Korourian S (2001) Plasma cells in chronic endometritis are easily identified when stained with syndecan-1. Mod Pathol 14(9): 877879.
23. Queen D, Perfetto C, Hazard F, Lathi R (2015) Pregnancy outcomes in women with chronic endometritis and recurrent pregnancy loss. Fertil Steril 104(4): 927-931.

24. Liu Y, Chen X, Huang J, Wang CC, Yu MY, et al. (2018) Comparison of the prevalence of chronic endometritis as determined by means of different diagnostic methods in women with and without reproductive failure. Fertil Steril 109(5): 832-839.

25. Kitaya K, Yasuo T (2013) Inter-observer and intra-observer variability in immunohistochemical detection of endometrial stromal plasmacytes in chronic endometritis. Exp Ther Med 5(2): 485-488.

26. Kasius JC, Broekmans FJ, Sie DM, Bougain C, Eijkemans MJ, et al. (2012) The reliability of the histological diagnosis of endometritis in asymptomatic IVF cases: a multicenter observer study. Hum Reprod 27(1): 153-158.

27. Yang R, Du X, Wang Y, Song X, Yang Y, et al. (2014) The hysteroscopy and histological diagnosis and treatment value of chronic endometritis in recurrent implantation failure patients. Arch Gynecol Obstet 289(6): 1363-1369.

28. Zargar M, Ghafourian M, Nikbakht R, Hosseini VM, Choghakabodi PM (2019) Evaluating chronic endometritis in women with recurrent implantation failure and recurrent pregnancy loss by hysteroscopy and immunohistochemistry. J Minim Invasive Gynecol pp. S1553-4650.

29. Polisseni F, bambirra EA, Camargos AF (2003) Detection of chronic endometritis by diagnostic hysteroscopy in asymptomatic infertile patients. Gynecol Obstet Invest 55(4): 205-210.

30. Song D, Li TC, Zhang Y, Feng X, Xia E, et al. (2019) Correlation between hysteroscopy findings and chronic endometritis. Fertil Steril 111(4): 772-779.

31. Kitaya K, Matsubayashi H, Takaya Y, Nishiyama R, Yamaguchi K, et al. (2017) Live birth rate following oral antibiotic treatment for chronic endometritis in infertile women with repeated implantation failure. Am J Reprod Immunol 78(5).

32. Vitagliano A, Saccardi C, Noventa M, Sardo AS, Saccone G, et al. (2018) Effects of chronic endometritis therapy on in vitro fertilization outcome in women with repeated implantation failure: a systematic review and meta-analysis. Fertil Steril 110(1): 103-112.

33. Kimura F, Takebayashi A, Ishida M, Nakamura A, Kitazawa J, et al. (2019) Review: Chronic endometritis and its effect on reproduction. J Obstet Gynaecol Res 45(5): 951-960.

34. Cicinelli E, Matteo M, Tinelli R, Pinto V, Marinaccio M, et al. (2014) Chronic endometritis due to common bacteria is prevalent in women with recurrent miscarriage as confirmed by improved pregnancy outcome after antibiotic treatment. Reprod Sci 21(5): 640-647. 